\title{
Evaluación de tres diluyentes naturales para semen fresco de conejo en la inseminación artificial
}

Evaluation of three natural diluents for fresh rabbit semen in artificial insemination

Rosero Peñaherrera Marco Antonio*, Núñez-Torres Oscar Patrício, Lozada-Salcedo Euclides Efraín

\begin{tabular}{l}
\hline DatoS del Artículo \\
\hline Universidad Técnica de Ambato. \\
Facultad de Ciencias Agropecuarias. \\
Cantón Cevallos. \\
Tungurahua - Ecuador. \\
Casilla postal: 18-01-334. \\
Telf: (+593)032872630-0985471191 \\
op.nunez@uta.edu.ec \\
ee.lozada@uta.edu.ec \\
*Dirección de contacto: \\
Universidad Técnica de Ambato. \\
Facultad de Ciencias Agropecuarias. \\
Cantón Cevallos. Tungurahua-Ecuador. \\
Casilla postal: 18-01-334. \\
Telf: (+593)032872630-0985471191 \\
Marco Antonio Rosero Peñaherrera \\
E-mail address : ma.rosero@uta.edu.ec \\
\hline
\end{tabular}

\section{Palabras clave:}

Semen,

diluyentes naturales,

viabilidad, motilidad,

concentración,

leche descremada

agua de coco,

aloe vera,

yema de huevo.

\section{J. Selva Andina Anim Sci.} 2018;5(1):23-32.

\section{Key words: \\ Semen, \\ natural diluents, \\ viability, \\ motility, \\ concentration, \\ skim milk. \\ coconut water \\ aloe vera, \\ egg yolk.}

Historial del artículo.

Recibido marzo, 2017.

Devuelto diciembre 2017

Aceptado febrero, 2018.

Disponible en línea, abril, 2018.

\begin{tabular}{c} 
Editado por: \\
Selva Andina \\
Research Society \\
\hline
\end{tabular}

Resumen

La presente investigación se ha realizado con el fin de evaluar la efectividad de diluyentes naturales empleados en el tratamiento de semen fresco de conejo (Oryctolagus cuniculus) en inseminación artificial y su utilidad en el campo de trabajo. Se han empleado tres diferentes tratamientos: leche descremada, aloe vera + yema de huevo y agua de coco, estudiando su efecto sobre motilidad, concentración y viabilidad espermáticas y parámetros reproductivos en conejas inseminadas artificialmente. La motilidad y viabilidad fueron evaluadas antes y después de la dilución, a 30, 60, 90, 120 $\min$.

Se emplearon 3 conejos machos mestizos y 12 hembras mestizas, de los cuales se tomó un macho y cuatro hembras por tratamiento, las hembras nulíparas con dos celos positivos previos, con una edad de 6 meses y un peso aproximado de 3.5 $\mathrm{Kg}$ y los machos con una edad de 10 meses y un peso promedio de $4.5 \mathrm{Kg}$, distribuidos con un diseño de bloques completamente al azar (DBCA), con 3 tratamientos de 4 repeticiones y un animal por unidad experimental. Se empleó una dosis única de $0.5 \mathrm{~mL}$ para la inseminación artificial con semen fresco de conejo adicionado con los tres tipos de diluyentes respectivamente, a un tiempo de 30 min de realizada la dilución. El estudio se llevó a cabo en un recinto experimental de jaulas elevadas durante un tiempo de 45 días.

Las variables evaluadas fueron: viabilidad y motilidad espermáticas en tiempos de 30, 60, 90, 120 min, tasa de concepción y número de gazapos. El agua de coco muestra un mejor desempeño sobre la tasa de concepción, número de crías, además de la motilidad y viabilidad espermática hasta un tiempo de $120 \mathrm{~min}$. El aloe vera y la combinación de leche con yema de huevo muestran resultados similares sobre la tasa de concepción, variando el en número de crías y siempre por debajo del agua de coco.

(C) 2018. Journal of the Selva Andina Animal Science. Bolivia. Todos los derechos reservados.

Abstract

The present investigation has been carried out in order to evaluate the effectiveness of natural diluents used in the treatment of fresh rabbit semen (Oryctolagus cuniculus) in artificial insemination and its usefulness in the field of work. Three different treatments have been used: skimmed milk, aloe vera + egg yolk and coconut water, studying its effect on motility, sperm concentration and viability and reproductive parameters in artificially inseminated rabbits. Motility and viability were evaluated before and after dilution, at 30, 60, 90, $120 \mathrm{~min}$.

Three mestizo male rabbits and 12 crossbred females were used, of which one male and four females were taken per treatment, the nulliparous females with two previous positive jealousies, with an age of 6 months and an approximate weight of $3.5 \mathrm{Kg}$ and the males with an age of 10 months and an average weight of $4.5 \mathrm{~kg}$, distributed with a completely random block design (DBCA), with 3 treatments of 4 repetitions and one animal per experimental unit. A single dose of $0.5 \mathrm{~mL}$ was used for the artificial insemination with fresh rabbit semen added with the three types of diluents respectively, at a time of $30 \mathrm{~min}$ after the dilution was carried out. The study was carried out in an experimental room with elevated cages for a period of 45 days. The evaluated variables were: sperm viability and motility at times of 30, 60, 90, 120 min, conception rate and number of kits. The coconut water shows a better performance on the conception rate, number of offspring, in addition to motility and sperm viability up to a time of $120 \mathrm{~min}$. The aloe vera and the combination of milk with yolk of egg show similar results on the rate of conception, varying the number of offspring and always below the coconut water. 


\section{Introducción}

El conejo común (Oryctolagus cuniculus) constituye una de las principales especies en crianza familiar en la serranía del Ecuador; por lo tanto el uso y desarrollo de nuevas técnicas asociadas a la reproducción, alimentación y manejo ayudan a mejorar notablemente la producción de dicho animal y en consecuencia al desarrollo socio-económico de la población que se dedica a la crianza de este animal, así como también a fomentar la cultura gastronómica y ornamental de esta especie.

A los largo de los años se ha fortalecido la explotación de este animal tanto como alimento y sustento económico para familias que en el último censo (2003) fue de 515809 conejos a nivel nacional haciéndose necesario aplicar nuevas técnicas de producción; la aplicación de técnicas de inseminación artificial ha generado ventajas sanitarias, de manejo, económicas, así como en el campo de la selección y mejora genética. La obtención de semen no es muy diferente a la empleada con otras especies (bovinos, ovinos.), cada muestra debe ser evaluada para su posterior utilización, el proceso de evaluación del semen es esencial ya que no todos los eyaculados poseen calidad suficiente como para fecundar a una hembra. (Vega et al. 2012).

Se utiliza diversos diluyentes para el semen que pueden ser comerciales o naturales, siendo lo importante sus características de mantenimiento del esperma, deben cumplir dos objetivos aumentar el volumen del eyaculado sin afectar la calidad seminal para aumentar el número de inseminaciones y conservar la capacidad fecundante de los espermatozoides durante el mayor tiempo posible tiempo posible. (Bonet et al. 2009). El diluyente permite conservar las células espermáticas por su aporte nutritivo, efecto tampón, control de contaminación etc. Así, la presente investigación se realizó con el fin de evaluar la efectividad de diluyentes naturales empleados en el tratamiento de semen fresco de conejo (Oryctolagus cuniculus) y su utilidad en el campo de trabajo.

La inseminación artificial (IA) un método qué, el semen es obtenido del macho, se introduce en el tracto genital femenino mediante instrumentos, evitando el contacto entre animales. El uso de esta técnica permite aumentar el número de servicios por macho (Salamon et al. 1990. Es posible un fraccionamiento del semen colectado, logrando un mayor aprovechamiento del macho, acelerando el proceso de mejora genética ya que logra cubrir mayor número de hembras que en la monta natural. La preservación y dilución del semen favorece aún más la eficiencia de IA, y la optimización de los machos (Gil $\&$ Oliveira 2005. La obtención de semen no es muy diferente a la empleada con otras especies (bovinos, ovinos, y otros), cada muestra debe ser evaluada para su posterior utilización, este proceso, es esencial ya que no todos los eyaculados poseen calidad suficiente como para fecundar a una hembra. (Vega et al. 2012).

Se utilizan diversos diluyentes para el semen que pueden ser comerciales o naturales, siendo lo importante las características de mantenimiento del esperma, deben cumplir dos objetivos aumentar el volumen del eyaculado sin afectar la calidad seminal para aumentar el número de inseminaciones y conservar la capacidad fecundante de los espermatozoides durante el mayor tiempo posible tiempo posible. (Bonet et al. 2009). El diluyente permite conservar las células espermáticas por su aporte nutritivo, efecto tampón, control de contaminación etc. El objetivo de este estudio fue evaluar la efecti- 
vidad de tres diluyentes naturales empleados en el tratamiento de semen fresco de conejo en el proceso de inseminación artificial, su influencia sobre la calidad del semen y los parámetros reproductivos de las hembras inseminadas.

\section{Materiales y métodos}

Se preseleccionaron 12 conejos machos de ellos, se tomaron muestras de semen, se realizaron valoraciones macro y microscópicas, para obtener 3 mejores machos, y ser seleccionados para el experimento. La selección de los animales fue garantizada con registros de nacimiento, calendario sanitario del lugar de cría. Las características tomadas en cuenta para la selección de los machos fueron: animales con cabeza y patas robustas, descenso completo de testículos, peso entre 4.0 y $4.5 \mathrm{~kg}, 10$ meses de edad, sin patologías presentes, testículos sin lesiones.

Para las hembras se evaluaron animales con rasgos femeninos muy marcados, desarrollo vulvar completo, peso entre 3.0 y $3.5 \mathrm{~kg}$, seis meses de edad, número de pezones no menor de 8 , formando parte de camadas de nacimiento de 10 individuos como mínimo, dos celos positivos confirmados.

El alojamiento fue en jaulas de malla electro soldada con la pared posterior en común, elevadas a 1.0 $\mathrm{m}$ de altura sobre el piso, con medidas de $0.5 \mathrm{x} 0.5 \mathrm{x}$ $0.76 \mathrm{~m}$, bebederos automáticos tipo tetina.

Cortinas plásticas tipo lona en ventanas externas y como aislamiento térmico interno. A las unidades experimentales se les suministró alimentación mixta compuesta de alimento balanceado comercial por la mañana y forraje verde de alfalfa (Medicago sativa) por la tarde. Agua a voluntad en bebedero automático tipo tetina.
Las hembras fueron colocadas en las instalaciones 7 días antes del inicio de la experimentación con el fin de ayudar para la regulación del ciclo estral.

La aplicación de una dosis única de $0.2 \mathrm{~mL}$ de GnRh (Ovarelinl) mostró buen resultado en el aparecimiento de signos de celo a partir de $48 \mathrm{~h}$, Cisneros-Prado (2010) señala como parte fundamental de la técnica de IA en coneja es la inducción de la ovulación inmediatamente después del servicio. Trabajando con IA en conejos reportó que la aplicación intramuscular de factores liberadores de hormonas gonadotropinas $(\mathrm{GnRh})$ inducían satisfactoriamente la ovulación sin formar anticuerpos en sangre después de aplicarse varias veces en una misma coneja. Luego de su aplicación, se evidenciaron por la edematización, cambio de coloración vulvar de rosa pálido a azul-violeta, el reflejo de inmovilización ante el salto del macho detector de celo, pérdida de apetito y la extracción de lana. (Chard 1975)

La hora de la extracción del semen fue a las 14:00 h con el fin de garantizar la calidad y volumen seminal, que podría estar influenciado por horarios de alimentación y condiciones climáticas. Los machos seleccionados para la experimentación fueron tentados al salto $5 \mathrm{~min}$, antes de la extracción con la vagina artificial. Para la obtención de las muestras seminales se introdujo a la coneja en la jaula del macho. Cuando el macho realizó el salto, se colocó la vagina artificial con la mano libre entre la grupa de la coneja y el vientre del conejo. Una vez tomada la muestra de semen, se procedió a realizar la valoración macroscópica, se determinó, aspecto seminal por uniformidad, color, volumen de eyaculado mediante la graduación de tubos colectores. Así mismo se valoraron las características microscópicas del fluido seminal como motilidad espermática, concentración mediante la valoración en cámara de Neubauer ajustándose a $10^{6}$ espermatozoides por $\mathrm{mL}$, 
porcentaje de espermatozoides anormales, porcentaje de mortalidad mediante un frotis y tinción, las cabezas de espermatozoides muertos en el momento previo de la preparación del frotis se tiñen de rosa, no así los vivos. La motilidad, viabilidad fueron evaluadas antes y después de la dilución, a 30, 60, 90, $120 \mathrm{~min}$.

Esta primera evaluación seminal, sirvió como base para la selección de los conejos machos cuyo semen presentó optimas características macroscópicas y microscópicas. El análisis de muestras seminales de los machos fue realizado cuatro días antes del proceso de inseminación artificial. La confirmación del celo positivo se llevó a cabo a las 12:00 h.

El procedimiento de IA con semen fresco se llevó a cabo con la recolección aséptica del eyaculado, luego la preparación del diluyente, la adición de la muestra y finalmente la inseminación en la hembra. Se utilizaron tres diluyentes naturales para el semen: Agua de coco, leche descremada y yema de huevo con aloe vera. El volumen de cada diluyente fue de 9 veces el volumen de eyaculado de cada reproductor. Se emplearon jeringuillas desechables y catéteres plásticos de $4 \mathrm{~cm}$ para la IA.

La dosis aplicada fue única, de $0.5 \mathrm{~mL}$ realizado a las 14:30 h con el fin de evitar las variaciones fisiológicas debido a los horarios de alimentación. La IA en la coneja fue puramente vaginal, se la realizó con catéteres plásticos semi-flexibles curvos y jeringuillas estériles. Se realizó la extracción de muestras en una coneja receptiva adulta para que el salto sea eficiente y se produzca la eyaculación completa en la vagina artificial.

Las muestras obtenidas de inmediato fueron sometidas a su medición de volumen y análisis de laboratorio para su posterior dilución. Cada muestra de cada macho fue valorada nuevamente para realizar la dilución y posterior inseminación a 12 conejas. Las unidades experimentales fueron distribuidas bajo un diseño de bloques completamente al azar (DBCA), con tres tratamientos y cuatro repeticiones, los diluyentes empleados en el experimento fueron: agua de coco, leche descremada y aloe vera + yema de huevo. El arreglo factorial fue de $3 * 4$, con un tamaño de la unidad experimental de 4 animales por tratamiento y un total del tamaño del experimento de 12 animales.

Se realizó el cálculo de la varianza con los resultados de las medias obtenidas de los tratamientos aplicados y con el fin de determinar el grado de significancia se aplicó la prueba estadística de Tukey al $0.05 \%$.

Con el software estadístico InfoStat se calculó la varianza de las variables dependientes, Concentración, Viabilidad, Motilidad espermáticas, tasa de concepción y número de gazapos, empleando las medias de los datos obtenidos.

Con los datos de la varianza se realizó la prueba de significancia de Tukey al $0.05 \%$ para conocer la significancia estadística a los cálculos realizados.

\section{Resultados}

Tabla 1 Volúmenes de diluyente y volúmenes totales de dilución de semen de conejo

\begin{tabular}{lccc}
\hline \multicolumn{1}{c}{ Diluyentes } & $\begin{array}{c}\text { Volumen de eyaculado } \\
(\mathrm{mL})\end{array}$ & $\begin{array}{c}\text { Volumen de diluyente } \\
(\mathrm{mL})\end{array}$ & $\begin{array}{c}\text { Volumen total de } \\
\text { Dilución }(\mathrm{mL})\end{array}$ \\
\hline $\begin{array}{l}\text { Agua de Coco } \\
\text { Leche descre- }\end{array}$ & 0.8 & 7.2 & 8.0 \\
mada & 2.0 & 18.0 & 20.0 \\
$\begin{array}{l}\text { Yema de huevo } \\
+ \text { Aloe vera }\end{array}$ & 2.6 & $23.4(11.7+11.7)$ & 26.0 \\
\hline
\end{tabular}


Tabla 2 Comportamiento reproductivo y evaluación espermática en conejos sometidos a la evaluación de tres diluyentes naturales en la inseminación artificial

\begin{tabular}{|c|c|c|c|c|c|c|}
\hline \multirow{2}{*}{ Índice } & \multirow{2}{*}{ Tiempo (min) } & \multicolumn{3}{|c|}{ Tratamientos } & \multirow[b]{2}{*}{ PROB. } & \multirow[b]{2}{*}{ C.V $(\%)$} \\
\hline & & $\mathrm{T}_{1}$ & $\mathrm{~T}_{2}$ & $\mathrm{~T}_{3}$ & & \\
\hline \multirow{4}{*}{ Viabilidad } & 30 & $0.78 \mathrm{a}$ & $0.84 \mathrm{~b}$ & $0.86 \mathrm{~b}$ & 0.0003 & 1.56 \\
\hline & 60 & $0.74 \mathrm{a}$ & $0.80 \mathrm{~b}$ & $0.79 b$ & 0.0018 & 1.77 \\
\hline & 90 & $0.71 \mathrm{a}$ & $0.76 b$ & $0.74 \mathrm{ab}$ & 0.0430 & 2.90 \\
\hline & 120 & $0.64 \mathrm{a}$ & $0.69 \mathrm{a}$ & $0.67 \mathrm{a}$ & 0.0625 & 3.54 \\
\hline \multirow{4}{*}{ Motilidad } & 30 & $0.96 \mathrm{~b}$ & $0.95 \mathrm{ab}$ & $0.93 \mathrm{a}$ & 0.0110 & 1.00 \\
\hline & 60 & $0.94 \mathrm{a}$ & $0.92 \mathrm{a}$ & $0.91 \mathrm{a}$ & 0.0787 & 1.65 \\
\hline & 90 & $0.92 b$ & $0.89 \mathrm{ab}$ & $0.88 \mathrm{a}$ & 0.0214 & 1.66 \\
\hline & 120 & $0.90 \mathrm{~b}$ & $0.80 \mathrm{a}$ & $0.78 \mathrm{a}$ & 0.0001 & 1.98 \\
\hline Tasa de concepción & & $1.00 \mathrm{a}$ & $0.75 \mathrm{a}$ & $0.75 \mathrm{a}$ & 0.4717 & 53.81 \\
\hline Tamaño camada & & $9.75 \mathrm{a}$ & $6.75 \mathrm{a}$ & $5.75 \mathrm{a}$ & 0.6699 & 52.92 \\
\hline
\end{tabular}

Figura 1 Viabilidad Espermática

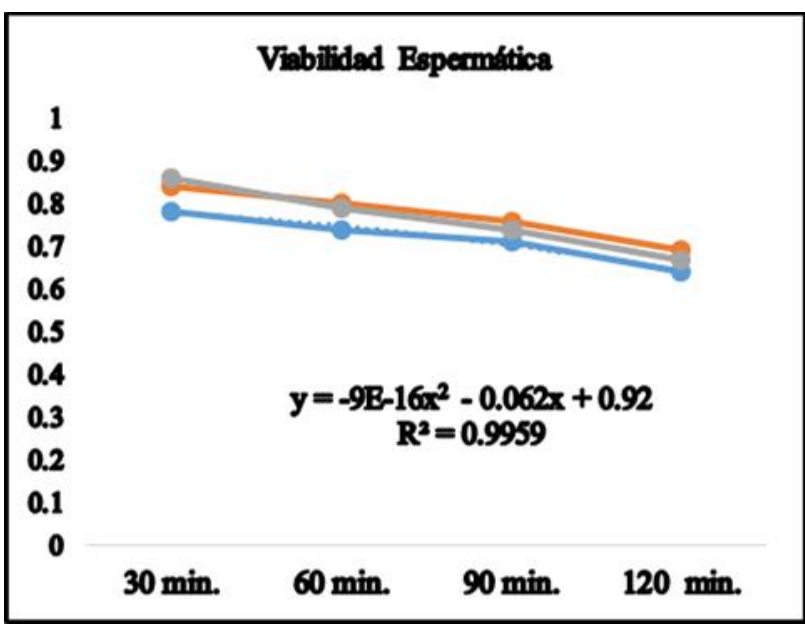

Figura 2 Motilidad Espermática

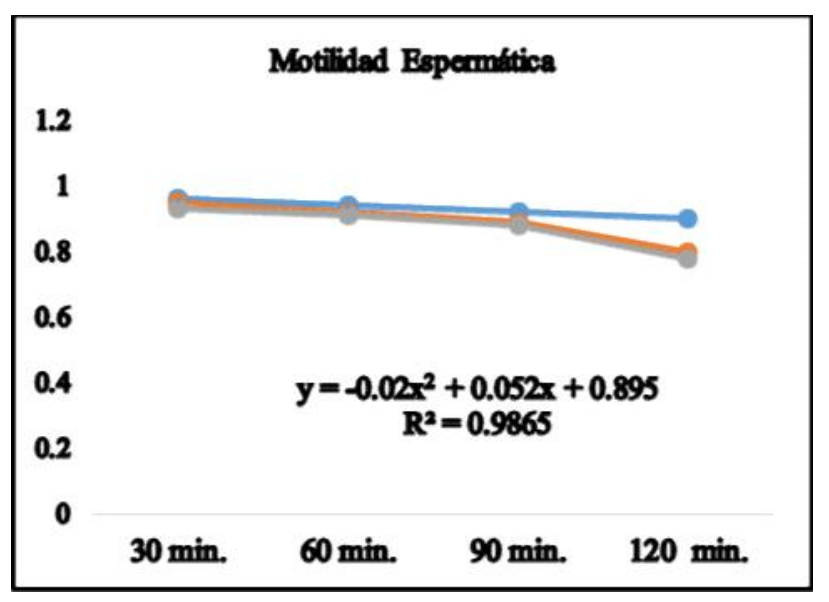

Figura 3 Tasa de Concepción

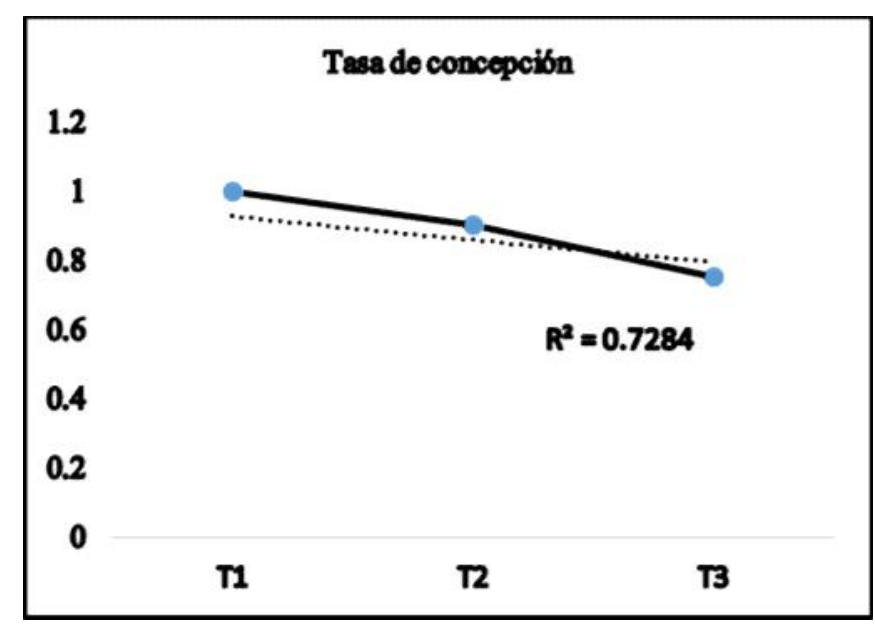

Figura 4 Tamaño de la Camada

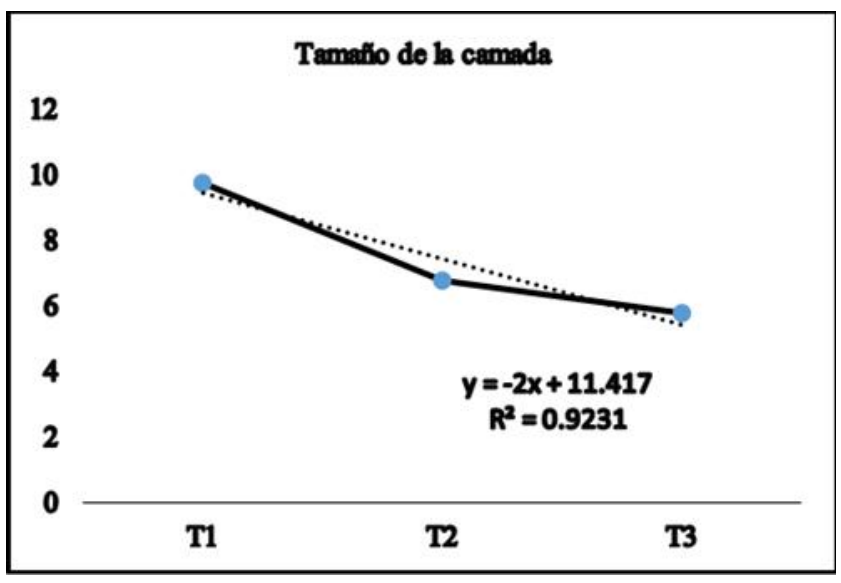




\section{Discusión}

Motilidad espermática. La motilidad espermática refleja la capacidad de movimiento idóneo que emplean los espermatozoides para poder alcanzar el objetivo de fecundar al óvulo; según Ferreira-Nunes (1993) la casi inexistencia de fosfolípidos en el agua de coco cuando se compara con la leche, no permite que las enzimas fosfolipasas presentes en las secreciones de las glándulas bulbouretrales, deterioren durante el metabolismo grandes cantidades de espermios, lo que se traduce en mayores probabilidades de sobrevivencia espermática concordando con lo obtenido en el experimento, resaltando que $\mathrm{T}_{1}$ obtuvo un mejor porcentaje de motilidad que $\mathrm{T}_{2} \mathrm{y}$ $\mathrm{T}_{3}$; a los 30 min no existe diferencia significativa con $\mathrm{T}_{2}$, pero sí con $\mathrm{T}_{3}$, siendo sus valores $\mathrm{T}_{1}(0.96)$, $\mathrm{T}_{2}(0.95)$ y para $\mathrm{T}_{3}(0.93)$.

Así mismo Aguilar-Jiménez (2015) indica que en un experimento con semen fresco de cerdo diluido con agua de coco la motilidad espermática fue de un $90 \%$ de vivos y un $10 \%$ de muertos a las $12 \mathrm{~h}$ y un $80 \%$ de vivos y un $20 \%$ de muertos a las 36 h post dilución, observándose similitud con los resultados obtenidos ya que a los 120 min de realizada la dilución $\mathrm{T}_{1}$ presenta un porcentaje de motilidad del $0.90 \%$ frente al $0.80 \%$ de $\mathrm{T}_{2}$ y $0.78 \%$ de $\mathrm{T}_{3}$, a su vez Ferreira-Nunes (1993) indica que la mayor sobrevivencia de los espermatozoides en el agua de coco, parece estar asociada a algunas sustancias que señala que son soluciones acidas y estériles, aminoácidos, azucares, sales, proteínas, vitaminas y minerales contenidas en la composición bioquímica del agua de coco, esa sustancia, es un factor físicoquímico que parece favorecer la motilidad del semen.

Viabilidad espermática. La capacidad de los espermatozoides de conservar las características funcionales, morfológicas y de capacitación se ven refle- jadas en la viabilidad, siendo esta una valoración muy importante al momento de realizar una inseminación artificial, concordando lo que señala Trejo et al. (2013) indicando que el uso de diluyente a base de agua de coco en congelación de semen tiene un efecto benéfico en la movilidad y viabilidad de los espermatozoides en relación con otros diluyentes naturales como es la leche descremada. A los 30 min la yema de huevo obtiene un $0.86 \%$ de viabilidad superando a $\mathrm{T}_{1}$ y $\mathrm{T}_{2}$ con valores de 0.78 y $0.84 \%$ respectivamente; a los 60 min $\mathrm{T}_{2}$ supera en viabilidad a los demás tratamientos con $0.80 \%$; a los 120 min de valoración la diferencia se acorta con $\mathrm{T}_{2}$ $0.69 \%$ frente a $\mathrm{T}_{1} 0.64 \%$ y $\mathrm{T}_{3} 0.67$ ya que la pérdida de viabilidad espermática es de $\mathrm{T}_{1}$ es de $0.14 \%$ frente a un $0.15 \%$ de $\mathrm{T}_{2}$ y $0.19 \%$ de $\mathrm{T}_{3}$ lo que indica que el semen fresco mantiene sus cualidades de viabilidad necesarias para un buen proceso de reproducción.

Tasa de concepción. La tasa de concepción corresponde al número de hembras gestantes de un total de hembras que estuvieron en celo en un tiempo determinado, $\mathrm{T}_{1}$ mostró tener una mejor tasa de concepción con el $100 \%$, frente al $0.75 \%$ de $\mathrm{T}_{2}$ y $\mathrm{T}_{3}$ respectivamente, concordando con Rodríguez de Lara et al. (2010) los cuales señalan que las tasas de concepción y la prolificidad en conejas receptivas inseminadas artificialmente e inducidas ovulatoriamente mediante factores liberadores de las hormonas gonadotropinas ( $\mathrm{GnRh}$ ) han mostrado ser altas, mientras que en las no receptivas sus comportamientos son bajos; mientras tanto que en estudios efectuados por Rebollar (1999) han confirmado el efecto positivo en la presentación de estros en conejas nulíparas sometidas a cambio de jaula $48 \mathrm{~h}$ antes de la inseminación y reportan tasas de concepción del $81.8 \%$.

Así mismo Trejo et al. (2013) emplearon como opción un diluyente a base de agua de coco obte- 
niendo resultados satisfactorios, tanto en el laboratorio, como en pruebas de IA en campo, con índices de fertilidad semejantes a los otros diluyentes tradicionales.

El tamaño de camada de conejas nulíparas puede variar entre 2 hasta 10 gazapos para el primer parto, dependiendo de la edad, raza, estación del año y condiciones generales del macho como de la hembra, según Trejo et al. (2013) la fertilidad observada utilizando semen fresco en la inseminación artificial fue $88 \%$ para el agua de coco y $80 \%$ para el MBO. Tamaño de la camada. Aguilar-Mejía 2015 manifiesta que uno de los aspectos importantes que resulta de la investigación con agua de coco como diluyente para semen fresco de cerdo es que no afecta el número de lechones nacidos vivos ya que se obtuvo un promedio de 10 lechones nacidos vivos por cerda y este es un buen indicador de efectividad, este dato fue importante en la elaboración del presente proyecto de tesis para ser empleado con semen fresco de conejo teniendo datos similares y muy aceptables.

Martín (1995) indica que aunque las conejas son susceptibles de ovular enseguida después del parto, la ovulación puede presentarse espontáneamente tan solo en un $17 \%$ de las hembras, influyendo sobre ello probablemente algunos factores de stress como la manipulación, la lactancia controlada, con una prolificidad de nacidos vivos de 8.7 gazapos por parto.

El número de gazapos obtenidos fue de 8.89 cuando se utilizó el agua de coco y 7.44 con el MBO ( $\mathrm{p}<0.05$ ), concordando con lo observado en la presente investigación, donde $\mathrm{T}_{1}$ obtuvo 9.75 gazapos de promedio frente a 6.75 de $\mathrm{T}_{2}$ y 5.75 de $\mathrm{T}_{3}$, lo que señala la superioridad del agua de coco para mantener las cualidades del semen fresco de conejo.
La mayor sobrevivencia de los espermatozoides en el agua de coco, parece estar asociada a algunas sustancias contenidas en la composición bioquímica del agua de coco, esa sustancia, es un factor físicoquímico que parece favorecer la motilidad del semen. Ferreira Nunes 1993.

Según Ferreira Nunes (1993) la casi inexistencia de fosfolípidos en el agua de coco cuando se compara con la leche descremada, no permite que las enzimas fosfolipasas presentes en las secreciones de las glándulas bulbouretrales de caprinos deterioren durante el metabolismo grandes cantidades de espermios, lo que se traduce en mayores probabilidades de sobrevivencia espermática concordando con lo obtenido en el experimento, resaltando que $\mathrm{T}_{1}$ obtuvo un mayor porcentaje de motilidad que $\mathrm{T}_{2} \mathrm{y}$ $\mathrm{T}_{3}$, a los $30 \mathrm{~min}$ no existe diferencia significativa con $T_{2}$, pero sí con $T_{3}$, siendo sus valores $T_{1}(0.96)$, $\mathrm{T}_{2}(0.95)$ y para $\mathrm{T}_{3}(0.93)$.

Así mismo Aguilar-Mejía 2015 indica que en un experimento con semen fresco de cerdo diluido con agua de coco la motilidad espermática fue de un $90 \%$ de vivos y un $10 \%$ de muertos a las $12 \mathrm{~h}$ y un $80 \%$ de vivos y un $20 \%$ de muertos a las 36 h post dilución, observándose similitud con los resultados obtenidos ya que a los 120 min de realizada la dilución $\mathrm{T}_{1}$ presenta un porcentaje de motilidad del $0.90 \%$ frente al $0.80 \%$ de $\mathrm{T}_{2}$ y $0.78 \%$ de $\mathrm{T}_{3}$, a su vez Ferreira Nunes (1993) indica que la mayor sobrevivencia de los espermatozoides en el agua de coco, parece estar asociada a algunas sustancias contenidas en la composición bioquímica del agua de coco; esa sustancia, es un factor físico-químico que parece favorecer la motilidad del semen.

La capacidad de los espermatozoides de conservar las características funcionales, morfológicas y de capacitación se ven reflejadas en la viabilidad, siendo esta una valoración muy importante al momento 
de realizar una inseminación artificial, concordando lo que señala Trejo et al. 2013, Gutierrez et al. 2013 indicando que el uso de diluyente a base de agua de coco en congelación de semen tiene un efecto benéfico en la movilidad y viabilidad de los espermatozoides en relación con otros diluyentes naturales como es la leche descremada. A los $30 \mathrm{~min}$ la yema de huevo obtiene un $0.86 \%$ de viabilidad superando a $\mathrm{T}_{1}$ y $\mathrm{T}_{2}$ con valores de 0.78 y $0.84 \%$ respectivamente, a los $60 \mathrm{~min} \mathrm{~T}_{2}$ supera en viabilidad a los demás tratamientos con $0.80 \%$, a los $120 \mathrm{~min}$ de valoración la diferencia se acorta con $\mathrm{T}_{2} \quad 0.69 \%$ frente a $\mathrm{T}_{1} 0.64 \%$ y $\mathrm{T}_{3} 0.67$ ya que la pérdida de viabilidad espermática es de $\mathrm{T}_{1}$ es de $0.14 \%$ frente a un $0.15 \%$ de $\mathrm{T}_{2}$ y $0.19 \%$ de $\mathrm{T}_{3}$ lo que indica que el semen fresco mantiene sus cualidades de viabilidad necesarias para un buen proceso de reproducción.

La tasa de concepción corresponde al número de hembras gestantes de un total de hembras que estuvieron en celo en un tiempo determinado, $\mathrm{T}_{1}$ mostró tener una mejor tasa de concepción con el $100 \%$, frente al $0.75 \%$ de $\mathrm{T}_{1}$ y $\mathrm{T}_{3}$ respectivamente, concordando con Fallas \& Rodríguez de Lara 1998 los cuales señalan que las tasas de concepción y la prolificidad en conejas receptivas inseminadas artificialmente e inducidas ovulatoriamente mediante factores liberadores de las hormonas gonadotropinas (GnRh) han mostrado ser altas, mientras que en las no receptivas sus comportamientos son bajos, mientras tanto que en estudios efectuados por Alvarino \& Rebollar (1995) han confirmado el efecto positivo en la presentación de estros en conejas nulíparas sometidas a cambio de jaula $48 \mathrm{~h}$ antes de la inseminación y reportan tasas de concepción del 81.8\%. El empleo como opción un diluyente a base de agua de coco obteniendo resultados satisfactorios, tanto en el laboratorio, como en pruebas de IA en campo, con índices de fertilidad semejantes a los otros diluyentes tradicionales.
El tamaño de camada de conejas nulíparas puede variar entre 2 hasta 10 gazapos para el primer parto, dependiendo de la edad, raza, estación del año y condiciones generales del macho como de la hembra, según Trejo et al. (2013) la fertilidad observada utilizando semen fresco en la inseminación artificial fue $88 \%$ para el agua de coco y $80 \%$ para el MBO. El número de gazapos obtenidos fue de 8.89 cuando se utilizó el agua de coco y 7.44 con el MBO ( $\mathrm{p}<0.05)$, concordando con lo observado en la presente investigación, donde $\mathrm{T}_{1}$ obtuvo 9.75 gazapos de promedio frente a 6.75 de $\mathrm{T}_{2}$ y $5.75 \mathrm{de}_{3}$, lo que señala la superioridad del agua de coco para mantener las cualidades del semen fresco de conejo. Por otro lado Martín (1995) indica que aunque las conejas son susceptibles de ovular enseguida después del parto, la ovulación puede presentarse espontáneamente tan solo en un $17 \%$ de las hembras, influyendo sobre ello probablemente algunos factores de stress como la manipulación, la lactancia controlada, con una prolificidad de nacidos vivos de 8.7 gazapos por parto.

A su vez Aguilar-Mejía 2015 manifiesta que uno de los aspectos importantes que resulta de la investigación con agua de coco como diluyente para semen fresco de cerdo es que no afecta el número de lechones nacidos vivos ya que se obtuvo un promedio de 10 lechones nacidos vivos por cerda y este es un buen indicador de efectividad.

El uso de diluyentes naturales para semen fresco de conejo en el proceso de la inseminación artificial mejora los índices productivos y la valoración seminal hasta los $120 \mathrm{~min}$.

El agua de coco muestra un mejor desempeño sobre la tasa de concepción, número de crías, además de la motilidad y viabilidad espermática hasta un tiempo de 120 min.

El aloe vera y la combinación de leche con yema de huevo muestran resultados similares sobre la tasa de 
concepción, variando el en número de crías y siempre por debajo del agua de coco.

Los procedimientos de crianza de animales en la actualidad han abierto infinidad de técnicas que ayudan a incrementar y mejorar la producción en las explotaciones de conejos, mismas que ayudan a conseguir mejores ingresos económicos en la realización de dicha actividad, como es el caso de cruces entre razas nuevas de animales, nuevos híbridos que van a aumentar la producción de carne o de crías, el adecuado estudio de técnicas de reproducción asistida como es el caso de la IA, las nuevas técnicas en la elaboración de alimento concentrado; las mismas que se encuentran contribuyendo al desarrollo de las técnicas de crianza de animales, ya sea para la industria los alimentos de origen animal o en el caso de animales de compañía.

El mejoramiento de las técnicas de reproducción animal conlleva el empleo de tiempo en el estudio de nuevas procedimientos y por lo tanto horas de esfuerzo de los productores, mismas que van a sr remuneradas con el mejoramiento de su producción, mismo que va destinado hacia un mercado cada vez más exigente en las explotaciones de conejos (Oryctolagus cuniculus) ornamentales, peleteras y de carne, en las cuales se espera obtener mayor cantidad de crías, animales de pesos adecuados, mayor belleza de sus mantos, etc., las cuales van a darle al productor un valor agregado de calidad y confianza por parte de su mercado.

La explotación del conejo (Oryctolagus cuniculus)en el medio artesanal en cualquiera de sus variedades no se encuentra tecnificada como es el caso de la producción industrial de cualquier otro tipo de animal, por lo que es necesario la implementación de técnicas utilizadas hace ya mucho tiempo en explotaciones industriales, y por si fuera poco el buscar un posible mejoramiento de las mismas, con 31 el fin de conseguir una mejor remuneración económica y con ello una mejor calidad de vida para el productor.

En el Ecuador, especialmente en la región sierra el conejo es uno de los platos más tradicionales y forma parte del sincretismo religioso, siendo tomado como una ofrenda para los dioses y en fiestas como el platillo principal. Los compuestos naturales de las plantas y las propiedades nutritivas de la leche y el huevo ayudan a la creación y evaluación de nuevos diluyentes para semen de distintas especies, especialmente de conejo. El mismo que en nuestro país no ha tenido un despunte sobre la tecnología empleada en la explotación del conejo.

\section{Conflictos de intereses}

Los autores declaran haber cumplido normas éticas en el manejo de los animales, el trabajo fue financiado por los autores y no genera conflictos de interés.

\section{Agradecimientos}

Los autores agradecen a la Universidad Técnica de Ambato, Facultad de Ciencias Agropecuarias, por el apoyo técnico, científico y logístico realizado a la presente investigación.

\section{Literatura citada}

Aguilar-Mejía JE. Evaluación del uso de agua de coco (Cocos nucifera 1.) como diluyente natural en inseminación artificial en cerdas. [Tesis Licenciatura]. Escuela De Zootecnia. Facultad De Medicina Veterinaria Y Zootecnia. Universidad De San Carlos De Guatemala; 2015. p. 48. 
Alvarino JMR, Rebollar PG. Control de la reproducción en cunicultura: tratamientos hormonales. Boletin de Cunicultura 1995;77:43-6.

Bonet S, Briz-González MD, Pinart E, Sancho S, Bussalleu-Muntada E, Yeste-Oliveras M, et al. Biotecnología de la reproducción porcina. Estado actual y futuro de las técnicas de análisis seminal. Anaporc 2009;6(63):18-23.

Chard RY. Los Conejos, Raza, Cría, Cuidados. Editorial de Vecchi, España; 1975.

Cisneros Prado MJ. Influencia de cambio de jaula y lactación controlada en conejas inseminadas artificialmente e inducidas a la ovulación con gonadorelina y fertirelina. [Tesis Maestría]. Universidad Autonoma Chapingo; 2010. p. 44.

Fallas LM, Rodríguez de Lara R. Sincronización de estros en conejas nulíparas mediante cambios de lugar y jaula y su efecto sobre el comportamiento reproductivo en inseminación artificial. Boletín de Cunicultura 1998;97:52-6.

Ferreira-Nunes J. El agua de coco como dilutor del semen caprino. Rev Fac Agron (Luz). 1993;3 (3):269-72.

Gil J, Olivera J. Preservación (Refrigeración y congelación) de semen ovino y su uso en la inseminación artificial cervical. XXXIII Jornadas Uruguayas de Buiatría, Paysandú, Uruguay; 2005. p. 55-67.

Gutiérrez AJ, Cosme RW, Jiménez CJA, Ramírez GJA. Agua de coco, suero fetal bovino, Aloe vera y sus combinaciones para criopreservar semen ovino. Arch Zootec 2006;55:101-4.

Martín M. Fisiología y Manejo Reproductivo de la Coneja. Universidad Autónoma de Barcelona. Cunicultura 1995;10(20):247-50.
Rebollar PG Últimos Avances en la Reproducción del Conejo. Departamento de producción animal. ETSI Agrónomos, Cdad. Universitaria s/n Madrid; 1999. (Disponible en: file:///C:/Users/ MAAC42 1.ROS/AppData/Local/Temp/Dialn et UltimosAvancesEnLaReproduccionDelConejo-2924204.pdf.

Rodríguez De Lara R, Fallas López M, Rangel Santos R, Mariscal Aguayo VD, Martínez Hernández PA, García Muñiz JG. Tecnología: 9. Programa práctico de inseminación artificial en conejos para granjas comerciales. Posgrado en Producción Animal, Departamento de Zootecnia, Universidad Autónoma Chapingo; 2010. p. 50-55. (Consultado el 5 septiembre del 2016). [Disponible en]: https://chapingo.mx/ produccionanimal/images/stories/documentos /Tecnologias/12-Tecnologia-9-IAconejos2008.pdf.

Salamon S, Maxwell WMC, Evans G. Inseminación artificial de ovejas y cabras. Zaragoza, Ed. Acribia; 1990. p. 192.

Trejo CA, Meza VVM, Antonio EC, Cotera RJ, Antonio-Cisneros CM. Agua de coco (cocus nucifera) como diluyente para semen fresco de conejo en la Inseminación Artificial. Arch Zootec 2013;62(238):299-302.

Vega MD, Barrio M, Quintela LA, Becerra JJ, Cainzos J, Prieto A, et al. Evolución del manejo reproductivo en cunicultura. Inf Téc Econ Agrar 2012;108(2):172-90. 